\title{
On Continuity and Boundedness of Fuzzy Syntopogenous Spaces
}

\author{
Zhiwen Mo ${ }^{1}$ Chang Shu ${ }^{2}$ \\ ${ }^{1}$ College of Mathematics and Software Science, Sichuan Normal University, Chengdu 610066, P.R.China \\ ${ }^{2}$ College of Information Management, Chengdu University of Technology, Chengdu 610069, P.R.China
}

\begin{abstract}
In this paper, we combine fuzzy topological structures with algebraic structures on $\mathrm{X}$, and investigate their corresponding structures and properties. In the section 3 , the relationship between fuzzy topological structure and increasing(decreasing) fuzzy syntopogenous structure is studied. In the section 4 the definition of continuity of a preordered L-fuzzy syntopogenous space $(X, S, \leq)$ and an example is given. In the section 5 the equivalent depictions of continuity are researched. In the section 6 the boundedness and its properties are discussed.
\end{abstract}

Keywords: Fuzzy topology, Algebra, Order, Continuity, Boundedness

\section{Introduction}

In [39] A.Csaszar introduced the concept of a syntopogenous structure to develop a unified approach to the three main structures of set-theoretic-topology: topologies, uniformities and proximities. This enable him to evolve a theory including the foundations of the three classical theories of topological spaces,uniform spaces and proximity spaces.In the case of the fuzzy structures there are at least three notions of fuzzy syntopogenous structures. The first notion worked out in $[4,5,6,12]$ presents aunified approach to the theories of Chang fuzzy topological spaces[1], Hutton fuzzy uniform spaces[2] and Liu fuzzy proximity spaces[7]. The second notion worked out in $[36,37]$ agree very well with Lowen fuzzy topological spaces[20], Lowen-Hohle fuzzy uniform spaces [17,21] and Artico-Moresco fuzzy proximity spaces[14]. The third notion worked out in [23] agree with the framework of a fuzzifying topology[25-28]. In [29], sostak introduced a new approach for a fuzzy topology as a fuzzy subset of the fuzzy powerset $\mathrm{I}^{\mathrm{X}}$ (i.e. a mapping $\left.\tau: \mathrm{I}^{\mathrm{X}} \rightarrow \mathrm{I}\right)$ satisfying certain axiom, the corresponding theory of fuzzy topological spaces containing Chang's approach as special, in a certain sense crisp case, was developed in a series of subsequent papers[29-35]. Based on the first notion of fuzzy syntopogenous structure the authors [9] established the general theory of syntopogenous structures on a completely distributive lattice and researched the unified question of cotopology, quasiuniformity and T-structure. In [10], we combined fuzzy topological structure on $\mathrm{X}$ with algebraic structure on $\mathrm{X}$, and investigated their corresponding structures and their properties. Concretely, we studied the preorder relation generated by an L-fuzzy syntopogenous structure. Conversely we researched the L-fuzzy syntopogenous structure defined by a preorder relation. And the increasing (decreasing) Lfuzzy syntopogenous space with preorder were defined, their properties were studied and an important example of an increasing L-fuzzy syntopogenous space $\left(R_{\phi}, S_{R}, \leq\right)$ was given. In this paper, we continue the research of [10]. In the section 3 , the relationship between fuzzy topological structures and increasing(decreasing)fuzzy syntopogenous structures is studied. In the section 4 the definition of continuity of a preordered L-fit syntopogenous space $(X, S, \leq)$ and two examples are given. In the section 5 the equivalent depictions of continuity are researched. In the section 6 the boundedness and its properties are discussed. In this paper we use notation, which is standard for the "fuzzy mexthematics", usually without explanation.

\section{Preliminaries}

In this paper, $L=<L, \leq, \wedge, \vee,^{\prime}>$ always denotes a completely distributive lattice with order-reversing involution "'"' . Let $\mathrm{O}$ be the least element and 1 be the greatest one in $\mathrm{L}$. Suppose $\mathrm{X}$ is a nonempty(usual)set, an L-fuzzy set in $\mathrm{X}$ is a mapping $A: X \rightarrow L$, and $L^{X}$ will denote the family of all L-fuzzy sets in $X$. It is clear that $L^{X}=<L^{X}, \leq, \wedge, \vee,^{\prime}>$ is a fuzzy lattice, which has the least element $\underline{0}$ and the greatest one $\underline{1}$, where $\underline{0}(x)=0, \underline{1}(x)=\underline{1}$, for any $x \in X$.

Definition 2.1.[4]) A binary relation $\ll$ on $L^{X}$ is called an L-fuzzy semi-topogenous order if it satisfies 
the following axioms : $(1) \underline{0}<<\underline{0}$ and $\underline{1}<<\underline{1}$; (2) $A<<B \quad$ implies $A \leq B^{-}$; (3) $A_{1} \leq A<<B \leq B_{1}$ implies $A_{1}<<B_{1}$. The complement of an L-fuzzy semi-topogenous order $<<$ is the L-fuzzy semi-topogenous order $<<^{c}$ defined by $A<<^{c} B$ iff $B^{\prime}<<A^{\prime}$. An L-fuzzy semitopogenous order $<<$ is called : (i)symmetrical if $<<=<<^{c}$; (ii)topogenous if $A_{1}<<B_{1}$ and $A_{2}<<B_{2} \quad$ imply $A_{1} \wedge A_{2}<<B_{1} \wedge B_{2} \quad$ and $A_{1} \vee A_{2}<<B_{1} \vee B_{2} \quad$; $\quad$ (iii)perfect if $A_{j}<<B_{j}, j \in J$ implies $\vee A_{j}<<\vee B_{j}$; (iv)coperfect if $A_{j}<<B_{j}, j \in J$ implies $\wedge \mathrm{A}_{\mathrm{j}}<<\wedge \mathrm{B}_{j}$; (v)biperfect if it is perfect and co-perfect.

Suppose that $<<_{1}, \quad<<_{2}$ are L-fuzzy semitopogenous orders on $X$, we call $<<_{1}$ finer than $<<_{2}$ ( i.e. $<<_{2}$ is coarser than $<<_{1}$ ) if for any $A, B \in L^{X}, \quad A<<_{2} B$ implies $A<<_{1} B$, it is denoted by $<<_{2} \leq<<_{1}$. If $<<_{1} \leq<<_{2}$ and $<<_{2} \leq<<_{1}$, then $<<_{1}=<<_{2}$. For a given L-fuzzy semi-topogenous order $<<$, we define $<<^{p},<<^{i},<<^{b}$ as follows : $A<<^{p} B$ iff there exist $A_{i}, i \in I$, such that $A=\vee A^{i}$, and for any $i, A_{i}<<B ; A<<^{i} B$ iff there exist $B_{j}, j \in J$ such that $B=\wedge B_{j}$ and $B<<B_{j}$ for any $j \in J ; A<<^{b} B \quad$ iff there exist $A_{i}$, $i \in I, B_{j}, j \in J$ such that $A=\vee A_{i}, B=\wedge B_{j}$ and $A_{i}<<B_{j}$ for any $i \in I, j \in J$.

Definition 2.2.([6]) An L-fuzzy syntopogenous structure on $X$ is a nonempty family $\mathrm{S}$ of L-fuzzy topogenous orders on $\mathrm{X}$ having the following two properties:(LFS1)S is directed in the sense that given any two members of $\mathrm{S}$ there exists a member of $\mathrm{S}$ finer than both ; (LFS2)for any $<<$ in $S$ there exists $<<_{1}$ in $\mathrm{S}$ such that $A<<B$ implies the existence of an $\mathrm{L}$ fuzzy set $C$ with $A<<_{1} C<<_{1} B$.

Let $S(X)$ be the set of all L-fuzzy syntopogenous structures on $X$. If $S$ is an L-fuzzy syntopogenous structure on $X$, then the pair $(X, S)$ is called an L-fuzzy syntopogenous space . An L-fuzzy syntopogenous structure $\mathrm{S}$ consisting of a single topogenous order is called a topogenous structure and the pair $(X, S)$ is called an L-fuzzy topogenous space. $S$ is called perfect (resp. co-perfect, biperfect) if each member of $\mathrm{S}$ is perfect(resp . co-perfect, biperfect). An L-fuzzy syntopogenous structure $S_{1}$ is called finer than another one $S_{2}$, if for each $<<$ in $S_{2}$ there exists a member of $S_{1}$ finer than $<<$. In this case we also say that $S_{2}$ is coarser than $S_{1}$, denoted by $S_{2} \leq S_{1}$. If $S_{1}$ is finer than $S_{2}$ and $S_{2}$ finer than $S_{1}$, then $S_{1}, S_{2}$ are called equivalent, denoted by $S_{1} \sim S_{2}$. To every L-fuzzy syntopogenous structure correspond two L-fuzzy topologies $\tau_{s_{0}} 、 \tau_{s}^{*}, \tau_{s}$ given by the interior operator $\mu^{0}=\sup \{\rho: \quad \rho<<\mu$ for some $<<\in S\}, \quad \tau_{s}^{*}$ given by the closure operator $\bar{\mu}=\wedge\{\rho: \mu \quad<<\rho$ for some $\quad<<\in S\}$. If $\left\{<<_{a}: a \in I\right\}$ is a family of L-fuzzy semi-topogenous orders on $\mathrm{X}$ then $<<=\vee_{a \in I}<<_{a}$ is the fuzzy semitopogenous order defined by $\mu<<\rho$ iff $\mu<<_{a} \rho$ for some $a \in I$. If $\mathrm{S}$ is a fuzzy syntopogenous structure, then it is easy to see that $<<_{s}=\vee\{<<:<<\in S\}$ is an L-fuzzy topogenous order and than $\left\{<<_{s}\right\}$ is an L-fuzzy topogenous structure . Moreover, $\mu \in \tau_{s}$ iff $\mu<<_{s}^{p} \mu$ and $\mu \in \tau_{s}^{*}$ iff $\mu^{\prime}<<_{s}^{i} \mu^{\prime}$. To every L-fuzzy topology $\tau$ on $X$ corresponds a perfect L-fuzzy topogenous structure, $S_{\tau}=\{<<\}$ where $\mu<<\rho$ iff there exists $\sigma \in \tau$ with $\mu \leq \sigma \leq \rho$, and a co-perfect Lfuzzy topogenous structure $S_{\tau}^{*}=\{<<\}$, where $\mu<<\rho$ iff there exists $E^{\prime} \in \tau$ with $\mu \leq E \leq \rho$. Moreover, $\tau=\tau_{S_{c}}$ and $\tau=\tau_{S^{*}}^{*}$, conversely, to every perfect(or co-perfect) $\mathrm{L}$-fuzzy topogenous structure $S=\{<<\}_{*}$ corresponds the Lfuzzy topology $\tau=\tau_{\mathrm{s}}$ (or $\tau=\tau_{\mathrm{s}}^{*}$ ) where $\mu \in \tau_{\mathrm{s}}$ iff $\mu<<\mu$ (or $\mu^{\prime}<<\mu^{\prime}$ ). To two different L-fuzzy topologies correspond different perfect(or coperfect)L-fuzzy topogenous structures.

\section{Topology and preorder}

A preorder on $X$ is a binary relation " $\leq$ " on $\mathrm{X}$ which is reflexive and transitive. A preorder on $\mathrm{X}$ which is also anti-symmetric is called a partial order or simply an order. By a preordered (resp. an ordered) set we mean a set with a preorder (resp. a partial order) on it.

Definition 3.1.([3]) Let $(X, \leq)$ be a preordered set. $A \in L^{X}$ is called:

(i)increasing, if $x \leq y$ implies $A(x) \leq A(y)$; (ii)decreasing, if $x \leq y$ implies $A(y) \leq A(x)$; (iii)order convex, if $y \leq x \leq z$ implies $A(y) \wedge A(z) \leq A(x)$.

Definition 3.2. Let $(X, \leq)$ be a preordered set, define mappings $\mathrm{p}$, a, c: $L^{X} \rightarrow L^{X}$. as follows : for

$A \in L^{X}, x \in X, p(A)(x)=\vee\{A(y): y \leq x\}$; $a(A)(x)=\wedge\{A(y): x \leq y\} ; c(A)=p(A) \wedge a(A)$. Lemma 3.1.([10] theorem 3.1) Let $(X, S)$ be an Lfuzzy syntopogenous space, define a binary relation $\leq_{s}$ on $X$ as follows : for any $x, y \in X, x \leq_{s} y$ iff for $A \in L^{X},<<\in S, \lambda \in L, \lambda \neq 0$ and $y_{\lambda}<<A$ implies $x_{\lambda} \leq A$. Then " $\leq$ "is a preorder on $\mathrm{X}$, it is called the preorder generated by $\mathrm{S}$ on $\mathrm{X}$.

Lemma 3.2.([10] theorem 3.5) Let $(X, \leq)$ be a preordered set, define $<<$ on $\mathrm{L}^{\mathrm{X}}$ as follows : for any $A, B \in L^{X} \quad$. $A<<B \quad$ iff $\quad x \leq y \quad$ implies $A(y) \leq B(x)$, then $S_{\leq}=\{<<\}$is an L-fuzzy biperfect topogenous structure, and for any $x, y \in X, x \leq y$ implies $x \leq_{s \leq} y$. 
Definition 3.3.([10] Def 4.1) Let $(X, \leq)$ be a preordered set, $S$ be an L-fuzzy syntopogenous structure on $X$, then $(X, S, \leq)$ is called increasing (decreasing)if for $x, y \in X, x \leq y$ implies $x \leq_{s} y\left(y \leq_{s} x\right)$.

Lemma 3.3.([10] Theorem 4.3) $S^{\mu}\left(S^{1}\right)$ is the finest one of all increasing (decreasing)L-fuzzy syntopogenous structures which are coarser than $S$ on $S(X)$, where $S^{\mu}=\vee\left\{S^{\prime} \in S(X):\left(X, S^{\prime}, \leq\right)\right.$ increasing, $\left.S^{\prime} \leq S\right\}$;

$$
S^{1}=\vee\left\{S^{\prime} \in S(X):\left(X, S^{\prime}, \leq\right)\right. \text { decreasing , }
$$
$\left.S^{\prime} \leq S\right\}$.

Proposition 3.4. Let $(X, \tau)$ be an L-fuzzy topological space, then the set of increasing(decreasing) $\tau$-open set is an L-fuzzy topology on $X$, denoted by $\tau^{\mu}\left(\tau^{1}\right)$.

Proof . We prove Proposition 3.4 by [3] Proposition 3.2.

Theorem 3.5. Let $(X, \tau)$ be an L-fuzzy topological space , and $S_{\tau}^{*}=\{<<\} \quad, \quad$ then $\tau_{S^{* \mu}}^{*}=\tau^{\mu}, \tau_{s^{* l}}^{*}=\tau^{l}$.

Proof . If $A \in \tau_{S^{* \mu}}^{*}$, as $S_{\tau}^{* \mu} \leq S_{\tau}^{*}$ by [10] Proposition $2.1 \tau_{S_{\tau}^{* \mu}}^{*} \tau_{S_{\tau}^{*}}=\tau$, also by [10] Proposition $4.1{ }^{S_{\tau}^{*}},{ }_{{ }_{\tau}} S_{\tau}^{*} \leq S_{\leq}$, hence $A \in \tau, A^{\prime}<<_{\leq} A^{\prime}$, i.e. $A \in \tau$ and $x \leq y$ implies $A^{\prime}(y) \leq A^{\prime}(x)$ (i. e. A increasing). So we have completed the proof of $\tau_{S^{* \mu}}^{*} \leq \tau^{\mu}$. Conversely, if $B \in \tau^{\mu} \quad$ (i.e. $B \in \tau$ and $\mathrm{B}$ increasing), then $B^{\prime}<<_{\tau}^{*} B^{\prime}$ and by [10] Corollary $3.6 B^{\prime}<<_{\leq} B^{\prime}$, let $<<_{0}=<<_{\tau}^{*} 0<<_{\leq}$, as $<<_{0} \leq<<_{\leq},<<_{0} \leq<<_{\tau}^{*}$ by Proposition 3.5 [5], we know that $\left(\mathrm{X}, \mathrm{S}_{0}=\right.$ $\left.\left\{<<_{0}\right\}, \leq\right)$ is increasing, and $S_{0} \leq S_{\tau}^{*}$, thus $S_{0} \leq S_{\tau}^{* \mu} \quad$ But $B^{\prime}<<_{0} B^{\prime} \quad$ implies $B^{\prime}\left(\bigcup^{\tau}<<\right)^{i} B^{\prime}$, and by Proposition 2.1 [10]

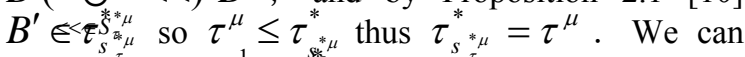
similarly prove $\tau^{1}=\tau_{\mathrm{s}^{*}{ }^{s_{2}}}$.

Let $(X, \delta)$ be an L-fuzzy proximity space (see [7]). by Proposition 2.2[10]. $S=\left\{<<_{\delta}\right\}$ is an Lfuzzy symmetrical_topogenous structure, where $A<<_{\delta} B$ iff $A \bar{\delta} B^{\prime}$. Let's define $\delta_{s^{\mu}}$ as follows: $A \overline{\delta_{s}{ }^{\mu}} \quad B$ iff $A<<B^{\prime}$, for some $<<\in S^{\mu}$. It is easy to prove that $\delta_{s^{\mu}}$ is an L-fuzzy proximity, denoted by $\delta^{\mu}$. We have the following $\delta^{\mu}$ depiction theorem.

Theorem 3.6. $A \delta^{\mu} B$ iff there exist an increasing Lfuzzy proximity $\delta_{\alpha}$ (i.e. $\left(X,\left\{<<_{\delta a}\right\}, \leq\right)$ increasing) which is coarser than $\delta$ and families of L-fuzzy sets $\left\{A_{i}: i \overline{\mathrm{mr}} 1, \ldots, m\right\}$ and $\left\{B_{j}: j=1, \ldots, n\right\}$ such that $\mathrm{A}=\vee \mathrm{A}_{\mathrm{i}}, \quad \mathrm{B}=\vee B_{j}^{\prime} \quad$ and $A_{i} \bar{\delta}_{a} B_{j}^{\prime}$ for $i \in\{1,2, \ldots, m\}, j \in\left\{1,2_{\hat{\lambda}}^{j=1} \ldots, n\right\}$.

Proof. As ${ }_{n} A \delta^{\mu} B$ iff $A<<B^{\prime}$ $\{<<\}=\left(S^{\mu}\right)^{t},\left\{r\left(U<<_{\alpha}\right)^{q}: a_{i} \in I\right\}=S^{\mu}, \quad$ so $A<<B^{\prime}$ iff $A\left(U^{i=1}<a_{a_{i}}\right)^{\alpha_{i}} B^{\prime}$, by Proposition 3.1[6] there exxist $\left\{\mathrm{A}_{1}, n_{n} \ldots, \mathrm{A}_{\mathrm{m}}\right\} \quad\left\{\mathrm{B}_{1}^{\prime}, \ldots, \mathrm{B}_{\mathrm{n}}^{\prime}\right\}$ and $A=\underset{i=1}{\vee} A_{i}, B^{\prime}=\wedge_{j=1} B_{j}$ for any $\mathrm{i}, \mathrm{j}$,
$A_{i}\left(\stackrel{n}{U}<<\alpha_{\alpha_{i}}\right) B_{j}$, thus there is $a_{i_{0}} \in I$ such that $A_{i} \stackrel{i=1}{<<} \alpha_{i} B_{j}$ i.e. $A_{i} \delta_{\alpha} \quad B_{j}^{\prime}$.

Theorem 3.7. Let $(X, \leq)$ be a preorder set, define a binary relation $<<_{0}$ on $L^{X}$ as follows: for any $A, B \in L^{X}, A<<_{0} B \quad$ iff $\quad x \leq y \quad$ implies $A(x) \leq A(y)$. Then $S_{\leq}^{*}=\left\{<<_{0}\right\}$ is an L-fuzzy biperfect topogenous structure, and for any $x, y \in X, \quad x \leq y$ implies $x \leq_{s^{*}} y$.

Proof. Please see the proof of theorem 3.5 [10].

Theorem 3.8. Let $(X, \leq)$ be a preordered set, $H_{i}=\left\{E \in L^{X}: E \quad\right.$ is increasing on $(X, \leq)\}, \quad H_{d}=\left\{E \in L^{X}: E\right.$ is decreasing on $(X, \leq)\}$. We define binary relations $<<_{H_{i}},<<_{H}$ as follows : $A<<_{H} B$ iff there exists $E \in H_{i}^{i}$ such that $A \leq E \leq B ; A<<H_{d} B$ iff there exists $E \in H_{d}$ such that $A \leq E \leq B$. Then(1) $<<_{H_{i}},<<_{H_{d}}$ are L-fuzzy biperfect topogenous orders; (2) $\left\{<<_{H_{i}}\right\}=S_{\leq}^{*},\left\{<<_{H_{d}}\right\}=S_{\leq}$.

Proof. (1)We can prove the results immediately by Proposition 3.2[3]. (2)As $A<<_{H_{i}} B$ iff there exists $E \in H_{i}$ such that $A \leq E \leq B$, , also $x \leq y$ implies $A(x) \leq E(x) \leq E(y) \leq B(y) \quad, \quad$ so $A(x) \leq B(y)$, thus $A<<_{0} B$, i.e. $\left\{<<_{H_{i}}\right\} \leq\left\{<<_{0}\right\}=S_{\leq}^{*}$.

Conversely , if $A<<_{0} B$, choose $E=p(A) \in H_{i}, \quad$ as $A<<_{0} B \quad$, so $x \leq y$ implies $A(x) \leq B(y)$, hence $A \leq p(A) \leq B$, thus $A<<_{H_{i}} B$, i.e. $\left\{<<_{0}\right\}=S_{\leq}^{*} \leq\left\{<<_{H_{i}}\right\}$. Therefore $\left\{<<_{H_{i}}\right\}=S_{\leq}^{*}$.

Similarly for $<<_{H_{d}}=S_{\leq}$.

Corollary 3.9. Let $\left(X,{ }^{d} \leq\right)$ be a preordered set, $S$ be an L-fuzzy syntopogenous structure on $X$, then $(X, S, \leq)$ is increasing iff $S \leq S_{H_{d}}=\left\{<<_{H_{d}}\right\}$.

Proof. We can prove the result immediately by Proposition 4. 2(3)[10].

\section{Definition of continuity}

A preordered (resp, an ordered) set $(X, \leq)$ on which there is a given L-fuzzy syntopogenous structure $\mathrm{S}$ is called a preordered (resp, an ordered) L-fuzzy syntopogenous space, denoted by $(X, S, \leq)$.

Definition 4.1. A preordered L-fuzzy syntopogenous space $(X, S, \leq)$ is called continuous iff for every $<<\in S$, there exists $<<_{1} \in S$ such that $A<<B$ implies $p(A)<<_{1} p(B)$ and $a(A)<<_{1} a(B)$.

Example 4.1. Any L-fuzzy syntopogenous space $(X, S)$ can be regarded as a preordered L-fuzzy syntopogenous space $(X, S,=)$ where "=" is the relation of the equality on $X$. Such space is always continuous.

Example 4.2. Let $(X, \leq)$ be a preordered set, define binary relation $<<_{0}$ on $L^{X}$ as follows: for any 
$A, B \in L^{X}, A<<_{0} B \quad$ iff $\quad x \leq y \quad$ implies $A(x) \leq A(y)$, by Theorem 3. 7, $S_{\leq}^{*}=\left\{<<_{0}\right\}$ is an L-fuzzy biperfect topogenous structure. Then $\left(X, S_{\leq}^{*}, \leq\right)$ is continuous.

In fact , if $A<<_{0} B$ and $x \leq y$, then $A \leq B, z \leq x$ implies $z \leq y, \quad A(z) \leq B(z)$, therefore

$p(A)(x)=\vee\{A(z): z \leq x\} \leq p(B)(y)=\vee\{B(z):$ $z \leq y\} \quad, \quad p(A)<<_{0} p(B) \quad$. Similarly $a(A)<<_{0} a(B)$.

Theorem 4.1. Let $(X, \tau)$ be an L-fuzzy topological space, $S=\left\{<<_{\tau}\right\}$, then preordered L-fuzzy syntopogenous space $(X, S, \leq)$ is continuous iff for any $\mu \in \tau$ implies $p(\mu) \in \tau$ and $a(\mu) \in \tau$.

Proof. Sufficiency: for any $\mu \in \tau, \mu<<_{\tau} \mu$,

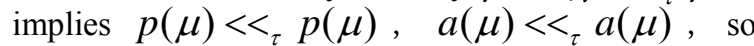
$p(\mu) \in \tau$ and $a(\mu) \in \tau$. Necessity: if $\mu \in_{\tau} \rho$, then there exists $\mu_{0} \in \tau$ such that $\mu \leq \mu_{0} \leq \rho$, so $p(\mu) \leq p\left(\mu_{0}\right) \leq p(\rho) \quad$ and $a(\mu) \leq a\left(\mu_{0}\right) \leq a(\rho), p\left(\mu_{0}\right) \in \tau, a\left(\mu_{0}\right) \in \tau$. hence $p(\mu)<<_{\tau} p(\rho)$ and $a(\mu)<<_{\tau} a(\rho)$.

Proposition 4.2. Let $(X, \delta)$ be an L-fuzzy proximity space([7]) , $S=\left\{<<_{\delta}\right\}$, then $(X, S, \leq)$ is continuous iff for every increasing (or decreasing) set $\rho \in L^{X}$, we have that $\rho(\mu) \delta \rho$ implies $\mu \delta \rho$ (or $a(\mu) \delta \rho$ implies $\mu \delta \rho)$.

The proof is omitted.

Proposition 4.3. Let $U$ be an L-fuzzy uniformity structure ([2])on $X, S_{u}$ (see[5]) is induced by $U$, then $\left(X, S_{u}, \leq\right)$ is continuous iff for every $u \in U$, there exists $u_{1} \in U$, such that $u(A) \leq B$ implies $u_{1}(p(A)) \leq p(B)$ and $u_{1}(a(A)) \leq a(B)$.

The proof is omitted.

\section{Definition of continuity}

Theorem 5.1. Let $(X, \leq)$ be a preordered set,$<<$ be an L-fuzzy semi-topogenous order on $X$, we define binary relations $<<^{\prime}$ and $<<^{\prime \prime}$ on $X$ as follows: $\mu<<^{\prime} \rho$ iff there exist $\mu_{1} \in L^{X}, \rho_{1} \in L^{X}$ such that $\mu_{1}<<\rho_{1} \quad$ and $\mu \leq a\left(\mu_{1}\right), a\left(\rho_{1}\right) \leq \rho, \mu<<<^{\prime \prime} \rho$ iff there exist $\mu_{1}, \rho_{1} \in L^{X} \quad$ such that $\mu_{1}<<\rho_{1}$ and $\mu<<p\left(\mu_{1}\right), p\left(\rho_{1}\right) \leq \rho$.

Then $<<^{\prime}$ and $<<^{\prime \prime}$ are L-fuzzy semi-topogenous orders on $X$ Let $p(<<)=<<^{\prime q}, a(<<)=<<^{\prime \prime q}$. If $S$ is an L-fuzzy syntopogenous structure on $X$, the $p(S)=\{p(<<):<<\in S\} \quad$ and $a(S)=\{a(<<):<<\in S\} \quad$ are L-fuzzy syntopogenous structures on $X$.

Proof. We can verify straightly from definition $2.1,2.2$ $c(S)$.
Proposition 5.2. Let $(X, \leq)$ be a preordered set, $<<$ be an L-fuzzy semi-topogenous order, then $p\left(<<^{p}\right) \leq p(<<)^{p}$, and $a\left(<^{p}\right) \leq a(<<)^{p}$, $c\left(<^{p}\right) \leq c(<<)^{p}$. And if $S$ is an L-fuzzy syntopogenous structure on $X$, then $p\left(S^{p}\right) \leq p(S)^{p}, a\left(S^{p}\right) \leq a(S)^{p}, c\left(S^{p}\right) \leq c(S)^{p}$ and $c\left(S^{t}\right) \sim c(S)^{t}$.

Then proof is omitted.

Proposition 5.3. If a preordered L-fuzzy syntopogenous space $(X, S, \leq)$ is continuous, then $\left(X, S^{p}, \leq\right),\left(X, S^{t}, \leq\right) \quad$ and $\quad\left(X, S^{t p}, \leq\right) \quad$ are continuous.

The proof is omitted.

Proposition 5.4. For every $\mathrm{j} \in \mathrm{J},\left(\mathrm{X}, \mathrm{S}_{\mathrm{j}}, \leq\right)$ is continuous, then $\left(X, \vee S_{j}, \leq\right)$ is continuous.

The proof is omitted

Theorem 5.5. Let $(X, S, \leq)$ be a preordered L-fuzzy syntopogenous space, then the following conditions are equivalent : (1) $(X, S, \leq)$ is continuous ; (2) $p(S) \leq S$ and $a(S) \leq S$; (3) $c(S) \leq S$; (4) $c(S)^{a} \leq S$, where $a \in\{t, p, b, t p\}$.

Proof. (1)iff(2). If $(X, S, \leq)$ is continuous, then for $<<\in S$, there exists $<<_{1} \in S$ such that $A<<B$ implies $p(A)<<_{1} p(B), a(A)<<, a(B) \quad, \quad$ hence $p(<<) \leq<<<_{1}^{q}=<<_{1}, a(<<) \leq<<<_{1}^{q}=<<<_{1}$. Conversel $\mathrm{y}$, if $p(S) \leq S$ and $a(S) \leq S$, then $<<\in S$, there exist $\quad<<_{1} \in S,<<_{2} \in S$ such that $p(<<) \leq<<$ and $a(<<) \leq<<<_{2}$, by Definition 2.2 there exists $\quad<<^{\prime} \in S \quad$ Such that $<<_{1} u<<_{2} \leq<^{\prime}$. Thus if $A<<B$, then $p(A) p(<<) p(B)$ and $a(A) a(<<) a(B) \quad, \quad$ so $p(A)<<^{\prime} p(B)$ and $a(A)<<^{\prime} a(B)$.

(2)iff (3) is obvious.

(3)iff (4). Because of $c(S) \leq c(S)^{a}$, but $c(S)^{a} \leq S, \quad$ so $c(S) \leq S$, conversely, if $c(S) \leq S$, then $c(S)^{a} \leq S^{a} \sim \mathrm{S}$.

Corollary 5.6. Let $(X, \leq)$ be a preordered set, $\tau$ be a topology on $X, S=\left\{<<_{\tau}\right\}$. Let $G_{p}=\{p(\mu): \mu \in \tau\}, G_{a}=\{a(\mu): \mu \in \tau\}$ $G_{c}^{p}=G_{a} \vee G_{p}$, the topology generated by the subbase $G_{p}$ (resp. $G_{a}, G_{c}$ ) is denoted by

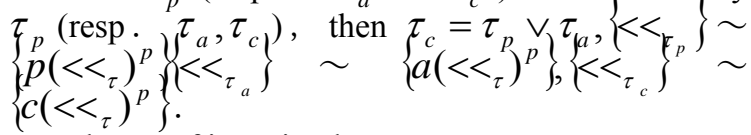
The proof is omitted.

Corollary 5.7. Let $(X, \delta)$ be an L-fuzzy proximity space, "s"be a preorder on $X, S=\left\{<<_{\delta}\right\}$, Let $\delta_{p}=\delta_{\left.p_{(<<\delta}\right)}, \delta_{a}=\delta_{q\left(<\delta_{\delta}\right)}$, then (1) $A \delta B$ iff $A \leq \wedge p\left(x_{j}\right), B \leq \vee \vee_{j=1} y_{j}$, where $x_{j} \delta y_{j}$, and $y_{j}$ is decreasing, $A, B, x_{j}^{j=1}, y_{j} \in L^{x}, 1 \leq j \leq n$. 
(2) $A \delta_{a} B$ iff $A \leq \wedge_{j=1}^{n} a\left(x_{j}\right), \quad B \leq \vee_{j=1}^{n} y_{j}$, where $\quad x_{j} \delta y_{j}$ and $y_{j}^{j=1}$ is increasing , $A, B, x_{j}, y_{j} \in L^{x}, 1 \leq j \leq n$.

The proof is omitted.

Corollary 5.8. Let $(X, \leq)$ be a preordered set, $\mathrm{U}$ be an L-fuzzy quasi-uniformity structure, let $p\left(S_{\mu}\right)^{b}=\left\{p(<<)^{b}: \quad<<\in S_{\mu}\right\}$, the L-fuzzy quasi-uniformity structure induced by $p\left(S_{\mu}\right)^{b}$ denoted by $\mu_{p} \quad$, similarly $\mu_{a}, \mu_{c}=\mu_{p} \vee \mu_{a}, S_{\mu_{p}}, S_{\mu_{c}}, S_{\mu_{a}} \quad$ then $S_{\mu_{\mathrm{p}}}=p\left(S_{\mu}\right)^{b} \quad, \quad S_{\mu_{a}}=a\left(S_{\mu}\right)^{b} \quad$ and $S_{\mu_{c}}=c\left(S_{\mu}\right)^{b}$.

The proof is omitted.

\section{Boundedness and its depictions}

Definition 6.1. A preordered L-fuzzy syntopogenous space $(X, S, \leq)$ is called bounded iff for every $<<\in S$, there exists $\varepsilon \subseteq L^{X}$ such that $A<<B$ implies there exists $C \in \varepsilon$ with $A \leq C \leq B$.

Theorem 6.1. If $(X, S, \leq)$ is totally bounded, then $(X, S, \leq)$ is bounded.

Proof. We can prove the result immediately by theorem 3.4[12].

Theorem 6.2. A preordered L-fuzzy syntopogenous space $(X, S, \leq)$ is bounded iff for every $<<\in S$, there exists $<<_{1} \in S$ and $\sigma \subseteq L^{x}$ such that $A<<B$ implies there exist $C, D \in \sigma \quad$ with $A \leq C \leq_{1} D \leq B$.

Proof. Necessity : We can prove the result by choose $\varepsilon=\sigma$.

Sufficiency : For $<<\in S$, by $\left(\mathrm{LFS}_{2}\right)$ there exists $<<_{1}$ with $<<\leq<<_{1} 0<<_{1} 0<<_{1}$. As $<<_{1} \in S$ and $(X, S, \leq)$ is bounded, So there is $\varepsilon \subseteq L^{X}$ with $A_{0}<<_{1} B_{0}$, therefore exists $C_{0} \in \varepsilon$ with $A_{0} \leq C_{0} \leq B_{0} \quad$. Now for $A<<B$, $<<\leq<<<_{1} 0<<_{1} 0<<<_{1} \quad, \quad$ so there exist $C, D \in L^{X}$ with $A<<_{1} C<<_{1} D<<_{1} B$. Thus there are $C_{1}, D_{1} \in \varepsilon$ such that $A \leq C_{1} \leq C$, $D \leq D_{1} \leq B \quad, \quad$ also $\quad C_{1} \leq C<<_{1} D \leq D_{1} \quad$ So $C_{1}<<_{1} D_{1}$. Above all, give $<<\in S$, there exist $<<_{1} \in S$ and $\varepsilon \subseteq L^{X}$ such that $A<<B$ implies there is $\quad C_{1}, D_{1} \in \varepsilon \quad$ satisfying $A \leq C_{1}<<_{1} D \leq B$, therefore the theorem is obtained.

Proposition 6.3. (1) If $S_{0}, S \in S(X), S \leq S_{0}$ and $S_{0}$ is bounded, then $S$ is bounded . (2)If $S \in S(X)$, then $S$ is bounded iff $S^{c}$ is bounded.

The proof is omitted.

Theorem 6.4. Suppose $f: x \rightarrow y$ is a mapping, $S^{\prime} \in S(Y)$ and $S=f^{-1}\left(S^{\prime}\right)$. (1)If $S^{\prime}$ is bounded, then $\mathrm{S}$ is bounded. (2)If $\mathrm{f}$ is onto and $S^{\prime}$ is bounded, then $\mathrm{S}^{\prime}$ is bounded.

Proof. (1)Suppose $<<^{\prime} \in S^{\prime},<<=f^{-1}\left(<<^{\prime}\right)$, by def. 6.1 there is $\varepsilon \subseteq L^{Y}$ such that $A_{0}<<^{\prime} B_{0}$ implies that there exists $C_{0} \in \mathcal{E}$ with $\mathrm{A}_{0} \leq \mathrm{C}_{0} \leq \mathrm{B}_{0}$.

$$
\text { Now suppose } A<<B \quad \text { so }
$$
$f(A)<<\left[f\left(B^{\prime}\right)\right]^{\prime}$, therefore for some $c \in \varepsilon$ such that $\quad f(A) \leq c \leq\left[f\left(B^{\prime}\right)\right]^{\prime} \quad, \quad$ and $A \leq f^{-1}(C) \leq B$.

(2)Suppose $\quad<<^{\prime} \in S^{\prime} \quad$ and $<<=f^{-1}\left(<<^{\prime}\right), \varepsilon \subseteq L^{X} \quad$ such that $A_{0}<<B_{0}$ implies there exists $C_{0} \in \varepsilon$ with $\mathrm{A}_{0} \leq \mathrm{C}_{0} \leq \mathrm{B}_{0}$. Now suppose $A<<B^{\prime}$, so $f^{-1}(A)<<f^{-1}(B)$, thus for some $c \in \mathcal{E}, f^{-1}(A) \leq \mathrm{C} \leq \mathrm{f}^{-1}(B) . \quad$ therefore $A \leq f(C) \leq B$.

Theorem 6.5. Suppose $S \in S(X), S^{\prime} \in S(Y)$ and $f:(X, S) \rightarrow\left(Y, S^{\prime}\right)$ is $\left(S, S^{\prime}\right)$-continuous onto mapping, If $S$ is bounded, then $S^{\prime}$ is bounded.

Proof. As $f$ is $\left(S, S^{\prime}\right)$-continuous, so $f^{-1}\left(S^{\prime}\right) \leq S$, therefore $f^{-1}\left(S^{\prime}\right)$ is bounded. By theorem 6.4(2) $S^{\prime}$ is bounded.

Theorem 6.6. $S \in S(X), S$ is bounded iff for every

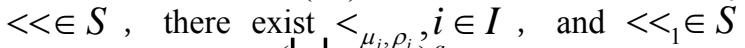
such that $<<\leq<<_{2}=\left(\bigcup<<{ }_{\mu_{i}, \rho_{i}}\right)^{q} \leq<<<_{1}$, where $<_{\mu_{i}, \rho_{i}}$ defined as fellows: $\quad A<_{\mu_{i}, \rho_{i}} B$ iff $A \leq \mu_{i} \leq \rho_{i} \leq B, \mu_{i}, \rho_{i} \in L^{X}$.

Proof. Sufficiency : For $<<\in S$, by theorem 6.2 , there exist $<<_{1} \in S$ and $\sigma \subseteq L^{x}$, set $<<_{2}=\left(\bigcup<_{\sigma_{1}, \sigma_{2}}\right)^{q}$ where $\left(\sigma_{1}, \sigma_{2}\right) \in \sigma \times \sigma$ and $\left.\sigma_{1}<\psi_{q_{1}} \sigma_{22}\right) \in \sigma \not d \mathrm{e}$ easily prove $<<\leq<<<_{2} \leq<<<_{1}$.

Necessity: For $<<\in S$, there is $<<_{1} \in S$ and $<<_{2}=\left(\bigcup<_{\mu_{y}, \rho_{i}}\right)^{q}$ such that $\leq<<_{2} \leq<<_{1}$. Set $\sigma=\left\{\mu \in L^{\gamma}, \rho^{\prime}, \mu=\underline{0}\right.$, or $\mu=\underline{1}$, or $\mu=\vee \wedge \mu_{m_{k j}}$ or $\mu=\vee \wedge \rho_{m_{k j}}$, where $\left.m_{k j} \in I\right\}$, We can prove $\sigma$ satisfying the condition. In fact, if $\mu<<\rho$, as $<<<<<_{2}$, so $\mu<<_{2} \rho$, therefore exist $A_{k}, B_{\mathrm{K}}, k=1,2,{ }_{\text {.Nk }}, K, j=1,2, \ldots N$ such that $\mu=\vee A_{k}, \rho=\wedge B_{j}, \quad A_{k}<<^{\prime} B_{j} \quad, \quad$ where $<<^{\prime}=\bigcup_{i \in I} \mathcal{C}_{\mu_{i}, p_{i}}{ }^{j=1}$ for $A_{k}<<^{\prime} B_{j}$, there exists $m_{k j} \in \in_{K} I, A_{k} A_{k}<\mu_{k} \mu_{m_{k j}}, \rho_{m_{k} K} B_{j}, A_{k} \leq \mu_{m_{k j}} \leq \rho_{m_{k j}} \leq B_{j} \Rightarrow$

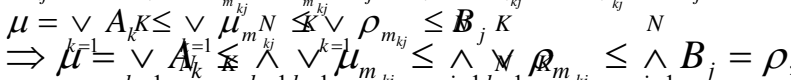

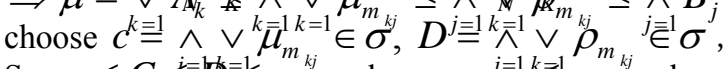

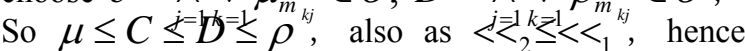
$\mu_{i}<<_{1} \rho_{i}, i \in I$, and $C<<_{1} D$, so the theorem is obtain.

Theorem 6.7. Suppose $\left(X, S_{\lambda}, \leq\right)_{\lambda \in \lambda}$ is a family of preordered L-fuzzy

Syntopogenous spaces. so $S=\vee_{\lambda \in \vee} S_{\lambda}$ is bounded $\Leftrightarrow$ for every $\lambda \in \wedge, S_{\lambda}$ is bounded.

Proof. If $\mathrm{S}$ is bounded, as $S_{\lambda} \leq S$, by proposition 6. 3(1) $S_{\lambda}$ is bounded. Conversely, for $<<\in S$, suppose $<<=\left(\bigcup_{i=1}<<_{\lambda_{i}}\right)^{q}$, where 
$<<_{\lambda_{i}} \in S_{\lambda_{i}}, \quad \lambda_{i} \in \wedge, \quad$ as $S_{\lambda_{i}}$ is bounded, order $\varepsilon_{\lambda_{i}} \subseteq L^{X^{\lambda_{i}}} \quad$ satisfying $A_{i}^{\lambda_{i}}<<_{\lambda_{i}} B_{i} \Rightarrow$ for some $C_{\lambda_{i}} \in \varepsilon_{\lambda_{i}}$ with $A_{i} \leq C_{\lambda_{i}} \leq B_{i}$. Now suppose $\mathrm{A}<<\mathrm{B} \quad, \quad$ so $\quad A=\vee_{j=1}^{m} A_{j}, B=\wedge B_{k} \quad$ and $A_{j}<<_{0} B_{k}$, wherre $<<_{0}=\bigcup_{i=1}^{n}<<_{i},{ }^{k=1}$ for $(\mathrm{j}, \mathrm{k})$ exists $C_{j k} \in \varepsilon_{m}=N \varepsilon_{\lambda_{i}}$ with $A_{j} \leq C_{j k} \leq B_{k}$, therefore $A \leq \vee \wedge=C_{j k} \leq B$. By the definition 6.1, we obtain the thềorèm.

\section{Acknowledgement}

This work is supported by National Nature Science Foundation of China (Grant No. 10671031), and partially Youth Science \& Technology Foundation of Sichuan Province (Grant NO. 07ZQ026-114).

\section{References}

[1] C. L . Chang, Fuzzy topological spaces, J. Math. Anal. Appl. 24 182-190, 1968.

[2] B. Hutton, Uniformities on fuzzy topological spaces, J. Math. Anal. Appl. 58:559-571, 1977.

[3] A. K. Katsaras, Ordered fuzzy topological spaces, J. Math. Anal Appl. 84:44-58, 1981.

[4] A . K . Katsaras, On fuzzy syntopogenous structures, Rev . Roumaine Math . Pures Appl. 30:419-431, 1985.

[5] A. K. Katsaras and C. G. Petalas, A unified theory of fuzzy topologies, fuzzy proximities and fuzzy uniformities , Rev. Roumaine Math. Pures Appl. 28:845856, 1983.

[6] A. K. Katsaras and C. G. Petalas, On fuzzy syntopogenous

J. Math. Anal. Appl. 99:219-236, 1984.

[7] Liu Wang-Jin , Fuzzy proximity spaces redefined, Fuzzy Sets and Systems 15:241-248, 1985.

[8] B. M. Pu and Y.M. Liu , Pointwise characterization of completely regularity and imbedding theorem in fuzzy topological space, SciSinica Ser A 26:138-147, 1983.

[9] Z. W. Mo and L. Shu, Syntopogenous structure on completely distributive lattice and its connectedness, Fuzzy Sets and Systems 72:365371, 1995.

[10] Z. W. Mo and L. Shu, On fuzzy syntopogenous structures and preorder, Fuzzy Sets and Systems, 90:355-359, 1997.

[11] L. A . Zadeh, Fuzzy Sets, Inform . and Control 8:338-353, 1965.
[12] A . K . Katsaras, Totally bounded fuzzy syntopogenous structures . Fuzzy sets and systems, 28:91-103, 1988.

[13] Z. W. Mo, On convexity of fuzzy Syntopogenous spaces, Fuzzy Sets and Systems 125:185-190, 2002.

[14] G. Artico and R. Moresco, Fuzzy proximities according with lowen fuzzy uniformities, Fuzzy Sets and Systems, 21:85-98, 1987.

[15] R. Badard, A. A. Ramadan and A. S. Mashhour, Smooth preuniform and preproximity spaces, Fuzzy Sets and Systems, 59, 1993.

[16] R. Badard, Smooth axiomatics, First IFSA Congress, plama de Mallorca.July, 1986.

[17] U. Hohle, Probabilistic metrization of fuzzy topologies. Fuzzy Sets and Systems, 1:345-356, 1978.

[18] U. Hohle and S. E. Rodabaugh, eds, Mathematics of Fuzzy Sets: Logic,Topology and Measure Theory,The handbooks of Fuzzy Sets Series,Volume 3(1999), Kluwer Academic Publishers(Boston/Dordrecht/London)

[19] U. Hohle, Many Valued Topology and Its Applications, Kluwer Academic Publishers (Boston/Dordrecht/London), March 2001.

[20] R. Lowen, Fuzzy topological spaces and fuzzy compactness, J.Math.Appl.56:,621-633, 1976.

[21] R. Lowen, Fuzzy uniform spaces, J.Math.Anal.Appl. , 82:,370-385, 1981.

[22] W. Peeters, Semi-pseudometric and pretopological methods in image analysis, Ph.D.thesis, Univ. Antwerpen, 2000.

[23] A. A. Ramadan, S. N. EI. Deeb, M-S. Saif and M. EI-Dardery, Fuzzifying syntopogenous structure, J.Fuzzy Math., 7:535-546, 1999.

[24] A. A. Ramadan, Smooth topological spaces, Fuzzy Sets and Systems,48, 1992.

[25] M. S. Ying, A new approach for fuzzy topology(I), Fuzzy Sets and Systems,39: 303-321, 1991.

[26] M. S. Ying, A new approach for fuzzy topology( II), Fuzzy Sets and Systems,47: 221232, 1992.

[27] M. S. Ying, A new approach for fuzzy topology(III), Fuzzy Sets and Systems,53:193207, 1993.

[28] M. S. Ying, A new approach for fuzzy topology, Fuzzy Sets and Systems, 53:93-104, 1993.

[29] A. P. Sostak, On a fuzzy topological structure, Suppl. Rend. Circ. Mat Palermo Ser. II, 11:89103, 1985.

[30] A. P. Sostak, On a compactness and connectedness degrees of fuzzy sets in fuzzy topological spaces In:general topology Relat, Modern Anal. and Algebra Proc. V. Prague 
Topol. Symp., Prague. (1986)Heldermann Verlag. Berlin.(1988).

[31] A. P. Sostak, On some modifications of fuzzy topologies, Mat Vesnik, 41:51-64, 1989.

[32] A. P. Sostak, On a category for fuzzy topology, Zb. Radove Univ. Nis, Ser. Matem. 2:61-67, 1988.

[33] A. P. Sostak, Two decades of fuzzy topology,basic ideas, notions and results.Russian Math. Surveys 44: 125-186, 1989.

[34] A. P. Sostak, On a neighhorhood structure of a fuzzy topological space, Zb. Radove Univ. Nis, Ser. Matem.4: 7-14, 1990.

[35] A. P. Sostak, On the convergence structure of a fuzzy topological space, In. proc. 2nd conf. Balcanic Union for fuzzy syst. Trabzon,Turkey, 1992.

[36] A. K. Katsaras, Fuzzy Syntopogenous structures compatible with lowen fuzzy uniformities and Artico-Moresco fuzzy proximities, Fuzzy Sets and Systems, 28:375-393, 1990.

[37] A. K. Katsaras, Operations on fuzzy syntopogenous structures, Fuzzy Sets and Systems, 47:199-218, 1991.

[38] Se Hwa chung, Separation axioms for fuzzy syntopogenous spaces, Fuzzy Sets and Systems, 101:463-468, 1999.

[39] A. Csaszar, Foundations of General Topology, Pergamon Press, 1963. 